\title{
Sulfonilureas, su uso actual en el tratamiento de la Diabetes Mellitus tipo 2
}

\author{
Sulfonylureas the present state in the tratment of Diabetes Mellitus type 2 \\ Rosa María Pando-Alvarez ${ }^{1}$
}

\section{Resumen}

Se hace una revisión actual de las sulfonilureas como hipoglicemiantes orales en el tratamiento de la diabetes mellitus tipo 2, particularmente de las sulfonilureas de segunda y tercera generación; asi como de su mecanismo de acción, riesgo cardiovascular, sus efectos adversos y su recomendación según las guías de la ADA, AACE y ALAD y su indicación en pacientes infectados con COVID-19.

Palabras clave: Diabetes Mellitus tipo 2, tratamiento, sulfonilureas.

\section{Abstract}

A current review of sulfonylureas as oral hypoglycemic agent in the treatment of diabetes mellitus type 2, particularly of the second and third generation sulfonylureas is performed. It is also reviewed its mechanism of action, cardiovascular risk, their adverse effects, the recommendation according to the ADA, AACE and ALAD guidelines and its use in patients infected with COVID-19.

Keywords: Diabetes Mellitus type 2, tratment, sulfonylureas.

\section{Introducción}

Las sulfonilureas (SU) son las primeras drogas hipoglicemiantes orales desarrolladas y utilizadas en el tratamiento de la diabetes tipo 2 (DM2). El descubrimiento de estos secretagogos de la insulina endógena, fue accidental; en 1942 Janbon y Loubatieres, al estudiar la sulfamida RP 2254 para el tratamiento de la fiebre tifoidea, observaron la aparición de convulsiones en algunos de sus pacientes, similares a las que se presentaban cuando había hipoglicemia inducida por altas concentraciones de insulina en animales de experimentación ${ }^{(1)}$.

Las SU se absorben en el tracto gastrointestinal, en el plasma, se encuentran unidas a proteínas en el 90 a $99 \%$, especialmente a la albúmina, son metabolizadas en el hígado y eliminadas por la orina y la bilis (Tabla 1).

Según la clasificación de la SU, las de primera generación actualmente ya no son utilizadas por tener una vida media prolongada y tener alto riesgo de hipoglicemia de gran duración. Las SU de segunda y tercera generación tienen una vida media menor y son prescriptas en el control de la diabetes en un $31 \%$ de casos en USA, en el Reino Unido en $45 \%$ y en otros países de Europa $^{(2)}$, su prescripción es mayor en la población latinoamericana $80 \%$, como en el Perú las SU se encuentran en el petitorio de fármacos a nivel nacional (Tabla 2).

El objetivo de esta revisión es establecer el papel actual del uso de las sulfonilureas como hipoglicemiante oral en el tratamiento de la DM2.

\section{Cuerpo de Trabajo}

\section{Las nuevas sulfonilureas}

A la segunda generación pertenecen la glipizida, glibenclamida o gliburide y la glicazida y a la de tercera

${ }^{1}$ Doctor en Medicina, Docente Principal Facultad de Medicina, Universidad Nacional Mayor de San Marcos, Lima-Perú. Instituto de Investigaciones Clínicas. ORCID: https://orcid.org/0000-0002-6112-802X 


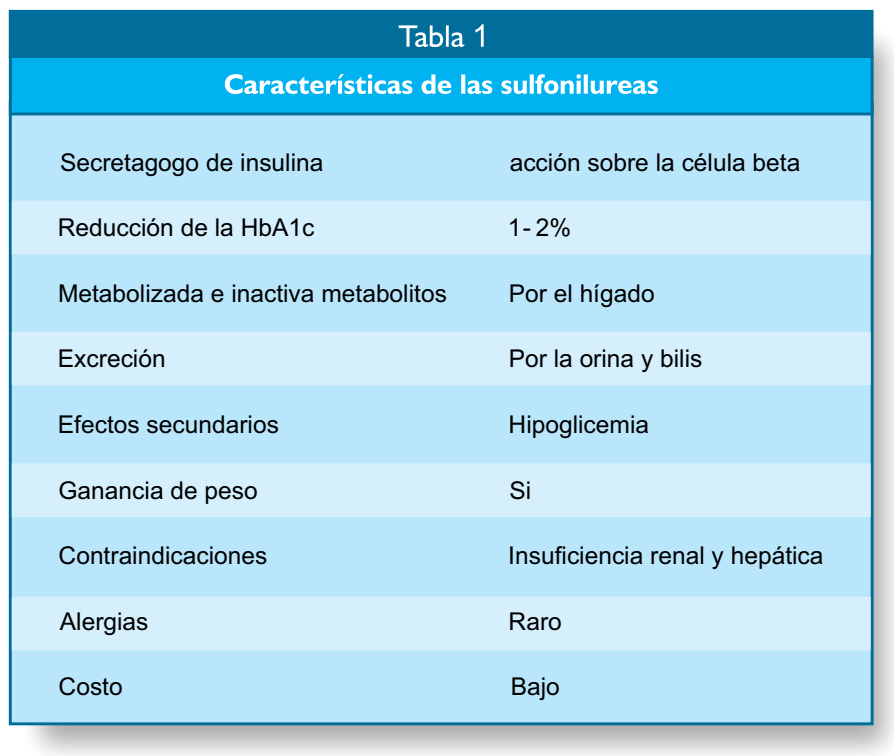

La Glimipiride la excreción en el 95\% es por las heces

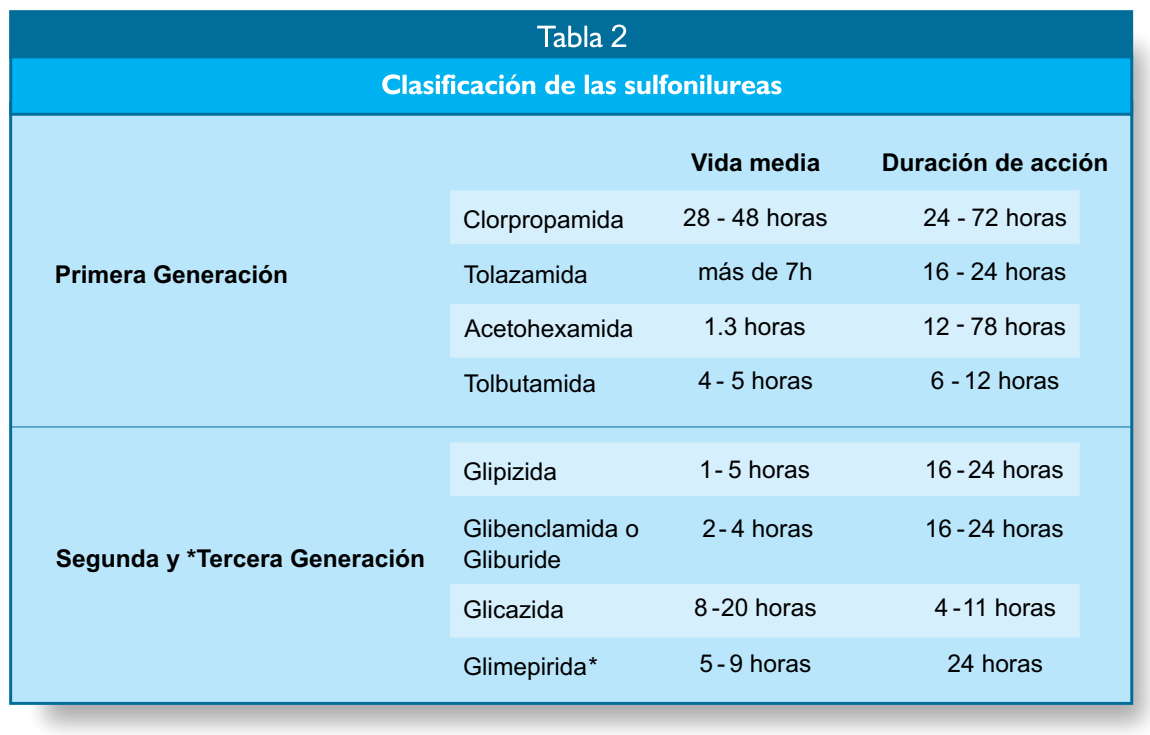

La glipizide, glicazida tienen presentaciones de liberación prolongada

generación la glimepirida. Tienen alta eficacia hipoglicemiante, reducen la hemoglobina glicosilada A1c (HbA1c) entre 1$2 \%$, se expende en menores concentraciones, tienen una vida media más corta que los de primera generación, tienen pocos efectos adversos e interacciones con otras drogas.

La glipizide y glicazida, que también se encuentran de presentación de liberación prolongada, son preferidos por ser administradas en una sola dosis al día, con bajo riesgo de hipoglicemia y menor ganancia de peso que la glibenclamida ${ }^{(3)}$. Se ha reportado que el control intensivo de la glucosa en la que se comparó la glicazida con otras drogas, con una reducción de la HbAlc $6.5 \%$ hubo una reducción de la nefropatía, complicación microvascular en el $21 \%$ y un efecto neutral sobre la complicación macrovascular ${ }^{(4)}$.

\section{Mecanismo de acción y el riesgo cardiovascular}

Su mecanismo de acción se basa en la liberación de insulina preformada y almacenada en la célula beta a la circulación, ante un estímulo desencadenado a partir del bloqueo del canal de potasio sensible al trifosfato de adenosina ATP- dependiente (K/ATP), al unirse al receptor de membrana de la célula beta (SUR1). 
La acción farmacológica se genera cuando la droga se une a un receptor de $\mathrm{SU}$, por lo que requiere de una célula beta funcionante para poder actuar. Los receptores SU-1 se localizan en las células beta pancreática y los receptores SU-2A y $\mathrm{B}$ se localizan en el músculo cardíaco y en el músculo liso vascular de arterias coronarias. Las SU deben tener alta afinidad al receptor SU-1 en la célula beta pancreática para evitar posibles interferencias en los mecanismos de adaptación a la isquemia (preacondicionamiento cardíaco, mecanismo endógeno por el cual se autoprotege el corazón de lesiones isquémicas letales). El preacondicionamiento ocurre cuando los canales (K/ATP) cardíacos se abren automáticamente durante episodios breves de isquemia miocárdica leve. Las $\mathrm{SU}$ que inhiben la apertura de los canales (K/ATP) cardíacos, por lo que pueden ser dañinas para el miocardio isquémico por supresión de los componentes dependientes del canal (K/ATP) de la respuesta de preacondicionamiento isquémico, mecanismo cardioprotector ${ }^{(5)}$.

Las SU de segunda generación como la glibenclamida o gliburide y tercera generación glimepirida carecen de selectividad para células $\beta$ pancreáticas que las SU glipizide y glicacida, lo que permite para que se unan a otros receptores, incluidos aquellos en cardiomiocitos y células vasculares del músculo liso (Figura 1). En el caso del gliburide, tal enlace ha sido sugerido para inhibir el preacondicionamiento isquémiisquémico ${ }^{(6-8)}$. Además, el gliburide y glimepirida producen metabolitos que son farmacológicamente activos y que podrían prolongar la duración de acción y tendría mayor riesgo de hipoglicemia ${ }^{(9)}$.

Varios estudios observacionales y metaanálisis han sugerido que particularmente la glibenclamida o gliburide tiene mayor riesgo cardiovascular al compararla con la glicazida ${ }^{(1)}$; sin embargo, se reconocen limitaciones metodológicas, incluidas clasificación de exposición errónea, intervalo de tiempo y el sesgo de selección que podrían afectar la validez de esos resultados.

Recientemente, el ensayo CAROLINA ha mostrado la seguridad cardiovascular de la glimepirida igual a la linagliptina inhibidor de dipeptidil peptidasa 4 (iDPP4) ${ }^{(12)}$.

Douros A. et al. ${ }^{(13)}$, reportaron que el gliburide y glimepirida de acción prolongada no se asociaron a un aumento de riesgo de infarto agudo de miocardio (HR 0,86; IC 0,551,34 ), accidente cerebrovascular isquémico (HR 0,92 ; IC 0,59 1,45 ), muerte cardiovascular (HR 1,01; IC $0,72-1,40$ ) y por cualquier causa mortalidad (HR 0,81 ; IC $0,66-1,003$ ), comparado con las SU de acción corta glipizida y glicacida,

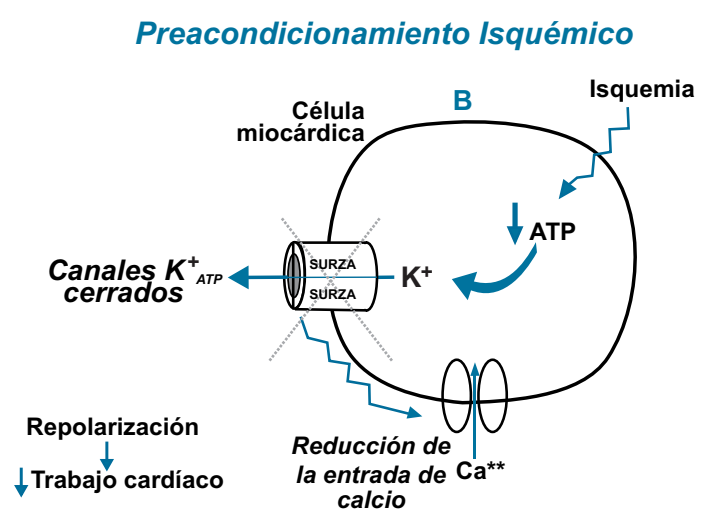

Sulfonilureas no selectivas. Acción en miocardio isquémico

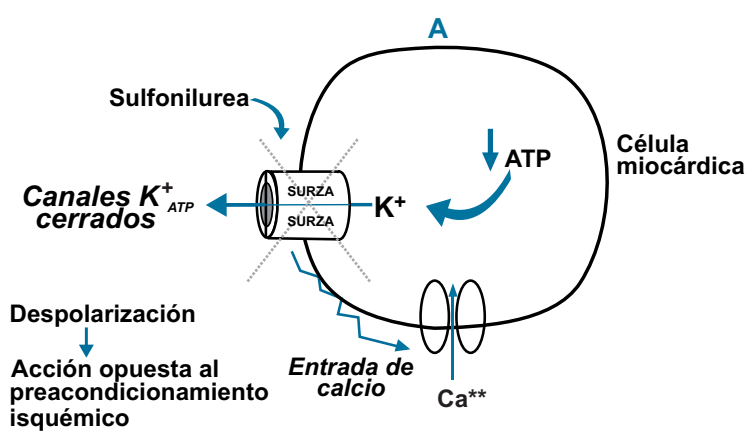

Figura 1. Sulfonilureas no selectivas y preacondicionamiento isquémico.

pero con un mayor riesgo de hipoglucemia grave (HR 2,83; IC $1,64-4,88)$ que podría contribuir a desencadenar eventos cardiovasculares.

La hipoglucemia ha sido implicada como un factor de riesgo para eventos cardiovasculares adversos y mortalidad por todas las causas en pacientes con diabetes ${ }^{(14)}$. En el estudio VADT (Veterans Affairs Diabetes Trial), se encontró que la hipoglucemia severa sería un predictor tanto de muerte cardiovascular y mortalidad por cualquier causa ${ }^{(15)}$ y el ensayo de evaluación de la acción en diabetes y enfermedades vasculares (ADVANCE) también mostró que la hipoglicemia severa podría contribuir a la presencia de eventos adversos cardiovasculares $^{(16)}$.

En el tratamiento prolongado las SU disminuyen su eficacia, al igual que otros hipoglicemiantes, esto se explicaría por la pérdida progresiva de la capacidad secretora de las células beta ${ }^{(17)}$, tanto debido a la historia natural de la diabetes como a la probabilidad de que estos agentes hipoglicemiantes aceleren la progresión de la falla de la célula beta. Estudios en humanos son difíciles de obtener porque solo están disponible aproximaciones indirectas de la masa de células beta. 
Antes del inicio de la intensificacion de la terapia con insulina, las $\mathrm{SU}$ mostraron un tiempo menor para mantener el control de la diabetes (6,3 años) que los agonista del GLP-1 (6,6 años) y los iDPP4 ( 7,1 años $)^{(18)}$.

\section{Efectos adversos. La hipoglicemia y ganancia de peso}

En estudios, como metaanálisis y ensayos, se ha mostrado que la glibenclamida se asocia con un mayor riesgo de hipoglicemia en comparación con la glicacida, la glimipiride y la glipizide; sin embargo, se consideró que el riesgo de hipoglicemia grave es relativamente pequeño ${ }^{(12)}$.

Recientemente, Dunkley AJ., et al. ${ }^{(19)}$ analizaron los eventos de hipoglicemia, la incidencia (eventos por persona/año) fue mayor para la insulina 4.39 y SU 2.34 que para la metformina 0.76 y para la incretina 0.56 , sin embargo, la incidencia de episodios graves fue similares entre que tomaron las SU (0.09), metformina (0.07) y las incretinas (0.07) como tratamiento para el control de la diabetes.

La dosis máxima comercializada es generalmente de dos a cuatro veces mayor que la dosis máxima efectiva. Para minimizar el riesgo de hipoglucemia, probablemente sea más apropiado emplear dosis relativamente bajas de SU con un cuidadoso monitoreo de la glucosa, se recomienda el uso de glicacida y glimepirida de liberación modificada que están asociadas a un mejor perfil de seguridad que la glibenclamida particularmente en adultos mayores o en pacientes con insuficiencia renal, insuficiencia hepática e insuficiencia cardíaca leve o moderada ${ }^{(20-23)}$. Si se quiere aumentar la acción hipoglicemiante se recomienda las combinaciones doble o triple de las SU con una dosis menor particularmente la glibenclamida con la metformina y las nuevas drogas como las incretinas o iDPP4, inhibidores del cotransportador sodio-glucosa tipo 2 ( iSGLT2) y así minimizar el riesgo de hipoglicemia ${ }^{(24-26)}$.

Otro efecto adverso es el aumento de peso, aproximadamente 2 a 4 kilos durante los primeros 3 o 4 años con el uso de SU, particularmente la glibenclamida en el estudio de UKPDS

${ }^{(27)}$. En el estudio Advance, el 90\% de participantes que estuvo tomando glicazida de liberación modificada, la ganancia de peso en el grupo intensivo fue $0.7 \mathrm{~kg}$ que el grupo de atención estándar ${ }^{(4)}$. En otro estudio a 5 años, al adicionar la metformina a la $\mathrm{SU}$, el aumento de peso medio fue $3,9 \mathrm{~kg}$ para gliclazida; $3,3 \mathrm{~kg}$ para glibenclamida; $0,1 \mathrm{~kg}$ para glimepirida ${ }^{(28)}$.

Para una menor ganancia de peso con las $\mathrm{SU}$, debe mantenerse el cambio de estilo de vida, adicionar la metformina al tratamiento con la SU o las nuevas drogas iDPP4 que tienen efecto neutro sobre el peso y se sugiere elegir la glimepirida que tiene una menor ganancia de peso que las demás SU.

\section{Las Guías}

Hasta el momento, las SU no se ha demostrado tener mayor riesgo cardiovascular comparado con las nuevas drogas orales como los iDPP4 11 y los iSGLT2, por lo que la han ubicado a las nuevas SU en drogas de segunda línea relativamente seguras y más populares después de la metformina en el tratamiento de la diabetes ${ }^{(29,30)}$.

Según la guía de la Asociación Americana de Diabetes (ADA 2020) ${ }^{(31)}$, cuando existe alto riesgo cardiovascular o enfermedad cardiovascular establecida o la presencia de enfermedad renal, así como necesidad de minimizar el riesgo de hipoglicemia o favorecer la pérdida de peso y si no se ha logrado la meta control $\mathrm{HbA} 1 \mathrm{c}<7 \%$, se recomiendan a la SU cómo última opción después de la metformina o análogos de los receptores de GLP-1 o iSGLT2 o iDPP-IV.

Al considerar los elevados costos de los nuevos fármacos hipoglicemiantes, la opción de la SU es la droga de segunda línea después de la metformina y si no se consigue meta de control de una $\mathrm{HbA} 1 \mathrm{c}<7 \%$, se considerará adicionar la insulina basal o iDPP4 o iSGLT2.

La guía de la Asociación Americana de Endocrinólogos Clínicos (AACE, 2020) a diferencia de la guía de la ADA, en los diabéticos sin comorbilidades serias y sin riesgo de hipoglicemia, considera como meta de control a una $\mathrm{HbAlc}$ $<6,5 \%$ y en aquellos con comorbilidades serias y riesgo de hipoglicemia, la meta control de la $\mathrm{Hb}$ Alc entre $6,5 \%$ y $7,5 \%$. Se recomienda la SU cómo monoterapia en última opción después de la metformina, GLP.1, iSGLT2, iDPP4, tiazolidinedionas (TZD) y la acarbosa.

La última guía de la Asociación Latinoamericana de Diabetes (ALAD, 2019), las SU es considerada una opción para reemplazar a la metformina en el caso de intolerancia, No recomiendan usarlo como monoterapia y evitar el uso de la glibenclamida en personas con diabetes y falla renal.

\section{Recomendaciones de su uso en pacientes infectados con el virus COVID-19}

Como en epidemias anteriores, la hiperglicemia ha sido un predictor independiente de severidad y mortalidad, es probable que también sea en el caso del COVID-19. La 
evidencia publicada en la literatura, muestra que el principal nexo entre diabetes y COVID-19 es de alto riesgo para una evolución más grave y mayor mortalidad ${ }^{(32)}$.

En un análisis de cohorte de 144 pacientes en el Reino Unido, diagnosticados con síndrome respiratorio agudo severo $(\mathrm{SARS})^{(32)}$ se encontró que el pobre control de la glicemia antes del ingreso al hospital se asoció con un alto riesgo de muerte durante la hospitalización. En pacientes DM2 con COVID-19 hospitalizados $^{(34)}$ con niveles de glucosa en sangre menores de $115 \mathrm{mg} / \mathrm{dl}$ tuvieron menor incidencia de linfopenia, neutrofilia $\mathrm{y}$ aumento en la proteína $\mathrm{C}$ reactiva y procalcitonina que los pacientes con niveles de glucosa en sangre de $\geq 135 \mathrm{mg} / \mathrm{dl}$. El buen control glucémico fue asociado con una menor tasa de complicaciones y todas las causas de mortalidad ${ }^{(34)}$. Li X et al. ${ }^{(35)}$, reportaron que la hiperglicemia durante el tratamiento en el hospital fue un factor de riesgo de muerte en pacientes con COVID-19 grave.

En la relación entre diabetes y COVID-19, se postula que las personas con diabetes tienen peor evolución debido tanto a las múltiples condiciones asociadas que aumentan el riesgo como por que el SARS-COV-2, debido a su tropismo por las células $\beta$ pancreática contribuiría al deterioro de la función de las células $\beta^{(36)}$ junto a la tormenta de citocinas inflamatorias y la respuesta hormonal contrarreguladora que pueden precipitar complicaciones metabólicas agudas (cetoacidosis o estado hiperosmolar) o el inicio de una diabetes en un paciente con COVID-19 $9^{(37-39)}$. Por lo cual se considera que la hiperglicemia al ingreso de la hospitalización y el deterioro metabólico agudo, a su vez, pueden empeorar aún más los resultados de COVID-19 ${ }^{(40)}$.

En el uso de hipoglicemiantes como las SU para el control de la diabetes en pacientes con COVID-19, se debe considerar algunos aspectos importantes. En una infección leve por COVID-19 y en un entorno ambulatorio, la terapia hipoglicemiante habitual que lleva la persona con DM2 podría continuar sin cambios, manteniendo una buena hidratación y aportando el requerimiento calórico adecuado, con un control más frecuente del monitoreo de la glucosa ${ }^{(41)}$. En pacientes DM2 que reciben tratamiento con cloroquina o hidroxicloroquina y/o una ingesta oral pobre en condición ambulatoria o hospitalizados por COVID-19 grave se recomienda modificar la terapia oral para la diabetes, particularmente los que reciben SU o insulina. El tratamiento con SU se debe suspender y reducir la dosis de insulina, debido al riesgo de hipoglicemia ${ }^{(42)}$.

El tratamiento con cloroquina o hidroxicloroquina puede causar hipoglucemia, particularmente en pacientes con insulina o SU, el mecanismo del efecto hipoglicemiante no es claro todavía $^{(43)}$. Se ha descrito que la cloroquina aumenta la respuesta del péptido $\mathrm{C}$, reflejando potencialmente una mejor función de las células beta pancreáticas ${ }^{(44)}$, reducción de la degradación insulina intracelular y el aumento de la acumulación de la insulina, posibles efectos de la hidroxicloroquina que han sido identificados en modelos animales ${ }^{(45)}$.

\section{Conclusiones}

El control de los pacientes con diabetes mellitus tipo 2 debe ser individualizado, la indicación de las SU debe ser por corto tiempo, en diabéticos con un tiempo de enfermedad no mayor de 5 años, mantenerse mientras tenga un buen control y sin factores de riesgo de hipoglicemia.

Se recomienda mantener el cambio de estilo de vida y la combinación con metformina o incretinas para la prevención de la ganancia de peso. En diabéticos con COVID-19 no se recomienda el uso de las SU cuando la condición clínica es grave y por la interacción con la hidroxicloroquina y el riesgo de hipoglicemia.

Las SU todavía están vigentes y son considerados como hipoglicemiantes de segunda línea en el tratamiento de la diabetes mellitus tipo 2 después de la metformina, particularmente en la mayoría de los países europeos y en la población latinoamericana, en la que influye de manera importante el costo de la medicación.

\section{Referencias bibliográficas}

1.Loubatieres A. The hypoglycemic sulfonamides: History and development of the problema from 1942 to 1955. Annals of the New York Academy of Sciences, 71: 4-11. doi:10.1111/j.17496632.1957.tb54569.x

2.Sharma M, Nazareth I, Petersen I. Trends in incidence, prevalence and prescribing in type 2 diabetes mellitus between 2000 and 2013 in primary care: a retrospective cohort study. BMJ Open 2016;6:e010210
3. Colagiuri S, Matthews D, Leiter LA, Chan SP, et al. The place of gliclazide MR in the evolving type 2 diabetes landscape: A comparison with other sulfonylureas and newer oral antihyperglycemic agents, Diabetes Res Clin Pract 2018;143:114.

4.Patel A, MacMahon S, Chalmers J, Neal B, et al. Intensive blood glucose control and vascular outcomes in patients with type 2 diabetes. N Engl J Med 2008,12; 358 (24):2560-272. 
5. Brady PA, Terzic A. The sulfonylurea controversy: more questions from the heart. J Am Coll Cardiol 1998;31(5):950956.

6. Riveline JP, Danchin N, Ledru F, Varroud-Vial M et al. Sulfonylureas and cardiovascular effects: from experimental data to clinical use. Available data in humans and clinical applications. Diabetes Metab 2003;29:207-222.

7. Abdelmoneim AS, Hasenbank SE, Seubert JM, Brocks DR, et al. Variations in tissue selectivity amongst insulin secretagogues: a systematic review. Diabetes Obes Metab 2012;14:130-138.

8. Gribble FM, Reimann F. Pharmacological modulation of K(ATP) channels. Biochem Soc Trans 2002;30:333-339.

9. Krentz AJ, Bailey CJ. Oral antidiabetic agents: current role in type 2 diabetes mellitus. Drugs 2005; 65:385-411.

10. Simpson SH, Lee J, Choi S, Vandermeer B, Abdelmoneim AS, Featherstone TR. Mortality risk among sulfonylureas: a systematic review and network meta-analysis. Lancet Diabetes Endocrinol 2015;3:43-51.

11. Abdelmoneim AS, Eurich DT, Gamble JM, Johnson JA, et al. Risk of acute coronary events associated with glyburide compared with gliclazide use in patients with type 2 diabetes: a nested case-control study. Diabetes Obes Metab 2014;16(1):22-29

12. Rosenstock J, Kahn SE, Johansen OE, Zinman B, et al. Effect of Linagliptin vs Glimepiride on Major Adverse Cardiovascular Outcomes in Patients with Type 2 Diabetes: The CAROLINA Randomized Clinical Trial. Jama 2019; 19:322(12):1155-1166.

13. Douros A, Yin H, Yu HY, Suissa S. Pharmacologic Differences of Sulfonylureas and the Risk of Adverse Cardiovascular and Hypoglycemic Events Diabetes Care 2017;40:1506-1513.

14. Paty BW. The Role of Hypoglycemia in Cardiovascular Outcomes in Diabetes Can J Diabetes 2015;39:S155-S159.

15. Hayward RA, Reaven PD, Wiitala WL, et al. VADT Investigators. Follow-up of glycemic control and cardiovascular outcomes in type 2 diabetes. N Engl J Med 2015;372:2197-2206.

16. Zoungas S, Patel A, Chalmers J, et al. For the ADVANCE Collaborative Group. Severe hypoglycemia and risks of vascular events and death. N Engl J Med 2010;363:1410-1418.

17. Kahn SE, Haffner SM, Heise MA, et al. Glycemic durability of rosiglitazone, metformin, or glyburide monotherapy. N Engl J Med 2006; 355:2427-2443.

18. Montvida O, Shaw J, Atherton JJ, Stringer F, Paul SK. Long-term Trends in Antidiabetes Drug Usage in the U.S.: Real-world Evidence in Patients Newly Diagnosed with Type 2 Diabetes. Diabetes Care 2018;41(1): 69-78.

19.Dunkley AJ, Fitzpatrick C, Gray LJ, Waheed G, Heller SR, Frier BM, et al. Incidence and severity of hypoglycaemia in type 2 diabetes by treatment regimen: a UK multisite 12 month prospective observational study. Diab Obes Metab 2019;21:1585-1595.

20. American Geriatrics Society Beers Criteria Update, Expert Panel. American Geriatrics Society 2019 Updated AGS Beers Criteria(R) for Potentially Inappropriate Medication Use in Older Adults. J Am Geriatr Soc. 2019;67(4):674-694.

21.Abdelmoneim AS, Eurich DT, Senthilselvan A, Qui W, et al. Dose-response relationship between sulfonylureas and major adverse cardiovascular events in elderly patients with type 2 diabetes. Pharmacoepidemiol Drug Saf 2016;25(10):1186-
1195.

22. Chan SP, Colagiuri S. Systematic review and meta-analysis of the efficacy and hypoglycemic safety of gliclazide versus other insulinotropic agents. Diabetes Res Clin Pract. 2015;110(1):75-81

23. Singh AK, Singh R. Is gliclazide a sulfonylurea with difference? A review in 2016.Expert Rev Clin Pharmacol. 2016;9(6):839-851.

24. Andersen SE, Christensen M. Hypoglycaemia when adding sulphonylurea to metformin: a systematic review and network meta-analysis. Br J Clin Pharmacol 2016;82(5):1291-1302

25. Kalra S, Bahendeka S, Sahay R, et al. Consensus recommendations on sulfonylurea and sulfonylurea combinations in the management of type 2 diabetes mellitus International Task Force. Indian J Endocrinol Metab. 2018;22(1):132-157.

26. Salvo F, Moore N, Arnaud M, Robinson P, et al. Addition of dipeptidyl peptidase-4 inhibitors to sulphonylureas and risk of hypoglycaemia: systematic review and meta-analysis. BMJ 201;3;353:i2231.doi :10.1136/bmj.i2231.

27. Intensive blood-glucose control with sulphonylureas or insulin compared with conventional treatment and risk of complications in patients with type 2 diabetes (UKPDS 33). UK Prospective Diabetes Study (UKPDS) Group. Lancet 1998;352:837-853.

28.Schrijnders D, Wever R, Kleefstra $\mathbf{N}$, et al. Addition of sulphonylurea to metformin does not relevantly change body weight: a prospective observational cohort study (ZODIAC39). Diabetes Obes Metab 2016;18: 973-979.

29. Winkler G. Sulfonylureas in today's blood glucose lowering therapy. New data on advantages and potential barriers of an "old" antidiabetic group. Orv Hetil 2015;29;156(13):511-515.

30. Webb DR, Davies MJ, Jarvis J, Seidu S, et al. The right place for Sulphonylureas today. Diabetes Res Clin Pract 2019; 157:107836 doi; 10.1016/j.diabres.2019.107836

31.American Diabetes Association. Pharmacologic Approaches to Glycemic Treatment: Standards of Medical Care in Diabetes 2020.Diabetes Care 2020;43(Suppl. 1):S98-S110.

32. Qiao S , Xiaoyi Z , Fang J , Xuanzhe Z, et al. Clinical Characteristics and Risk Factors for Mortality of COVID-19 Patients With Diabetes in Wuhan, China: A Two-Center, Retrospective Study. Diabetes Care 2020;43(7):1382-1391.

33. Booth CM. Clinical features and short-term outcomes of 144 patients with SARS in the greater Toronto area. JAMA 2003;289:2801-2809.

34.Zhu L, She ZG, Cheng X, et al. Association of blood glucose control and outcomes in patients with COVID-19 and preexisting type 2 diabetes. Cell Metab 2020;31:1068-1077.

35. Li X, Xu S, Yu M, et al. Risk factors for severity and mortality in adult COVID-19 inpatients in Wuhan. J Allergy Clin Immunol 2020; published online April. doi.org.10.1016/j. jaci.2020.04.006.

36. Hamming I, Timens W, Bulthuis MLC, Lely AT, et al. Tissue distribution of ACE2 protein, the functional receptor for SARS coronavirus. A first step in understanding SARS pathogenesis. J Pathol 2004;203:631-637.

37.Sobngwi E, Choukem SP, Agbalika F, et al. Ketosis-prone type 2 diabetes mellitus and human herpes virus 8 infection in sub-Saharan Africans. JAMA 2008;299:2770-776.

38. Bode B, Garrett V, Messler J, et al. Glycemic characteristics and clinical outcomes of COVID-19 patients hospitalized in the United States. J Diabetes Sci Technol 2020;14:813-821. 
39. Chee YJ, Huey Ng SJ, Yeoh E. Diabetic ketoacidosis precipitated by Covid-19 in a patient with newly diagnosed diabetes mellitus. Diabetes Res Clin Pract 2020;164:108166.

40. Apicella M, Campopiano MC, Mantuano M, Mzoni L, et al. COVID-19 in people with diabetes: understanding the reasons for worse outcomes www.the lancet.com/diabetesendocrinology Published online July, 2020 doi.org/10.1016/S2213-8587(20)30238-2.

41. Bornstein SR, Rubino F, Khunti K, et al. Practical recommendations for the management of diabetes in patients with COVID-19. Lancet Diabetes Endocrinol 2020;8:546-550.

42. Unubol M, Ayhan M, Guney E. Hypoglycemia induced by hydroxychloroquine in a patient treated for rheumatoid arthritis. J Clin Rheumatol 2011;17:46-47.
43. Rekedal LR, Massarotti E, Garg R, Bhatia R, Gleeson T, Lu B. Changes in glycosylated hemoglobin after initiation of hydroxychloroquine or methotrexate treatment in diabetes patients with rheumatic diseases. Arthritis Rheum. 2010;62(12):3569-3573.

44. Gerstein HC, Thorpe KE, Taylor DW, Haynes RB. The effectiveness of hydroxychloroquine in patients with type 2 diabetes mellitus who are refractory to sulfonylureas-a randomized trial. Diabetes Res Clin Pract 2002; 55(3):209219.

45. Emami J, Pasutto FM, Mercer JR, Jamali F. Inhibition of insulin metabolism by hydroxychloroquine and its enantiomers in cytosolic fraction of liver homogenates from healthy and diabetic rats. Life Sci. 1998;64(5):325-335.

Contribución de autoría: Rosa María Pando-Alvarez ha sido la autora del estudio, contribuyendo con concepción, búsqueda electrónica, revisión inicial, el diseño de estudio, redacción, y revisión final.

Conflicto de interés: La autora no tiene conflictos de interés con la publicación de este trabajo. Financiamiento: Autofinanciado.

Citar como: Pando-Alvarez RM. Sulfonilureas, su uso actual en el tratamiento de la Diabetes Mellitus tipo 2. Diagnóstico (Lima). 2020;59(1):16-22..

DOI: http://doi.org/10.33734/diagnostico.v59i1.203

Correspondencia: Rosa María Pando Alvarez. Correo electrónico: rpando21@gmail.com

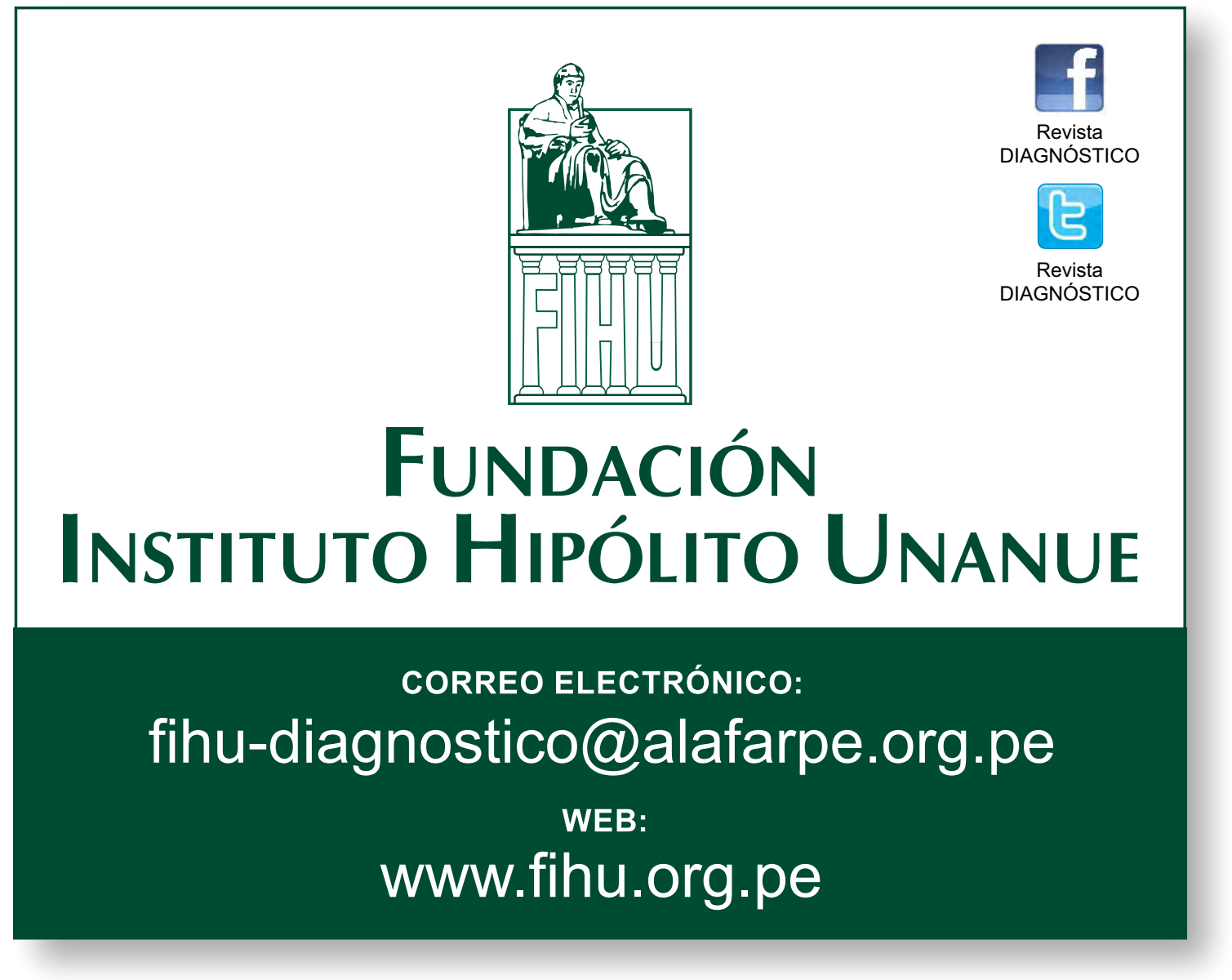

